\title{
Preditores clínicos do ganho estatural do primeiro ano de tratamento com dose fixa de hormônio de crescimento em crianças nascidas pequenas para idade gestacional
}

\author{
Clinical predictors of growth response in the first year \\ of treatment with a fixed dose of growth hormone \\ in children born small for gestational age
}

Adriana Claudia Lopes Carvalho Furtado', Luiz Claudio Gonçalves de Castro², Marcelo Palmeira Rodrigues ${ }^{3}$, Luciana Ansaneli Naves ${ }^{4}$

\section{RESUMO}

Objetivo: Identificar preditores clínicos associados à recuperação do crescimento no primeiro ano de tratamento com somatropina ( $\mathrm{rhGH}$ ) em um grupo de crianças nascidas pequenas para a idade gestacional (PIG). Sujeitos e métodos: Foram avaliadas 39 crianças que estavam em terapia com rhGH por pelo menos um ano $(0,33 \mathrm{mg} / \mathrm{kg} / \mathrm{semana})$. As variáveis analisadas foram idade cronológica (IC), idade óssea (IO), altura-alvo e escores de desvios-padrão (Z-escore) do peso e comprimento ao nascer, altura, peso e velocidade de crescimento pré e pós-rhGH. Resultados: A terapia com rhGH foi associada a incremento significativo de 0,67 Z-escore ( $p<$ $0,01)$ na estatura nesse primeiro ano. 10 e Z-escore de peso ao nascimento foram reconhecidos como preditores dessa resposta, com diferença significativa na variação do Z-escore de estatura entre pré-púberes e púberes $(p=0,016)$. Conclusão: Esses dados mostram ganho estatural significativo com dose fixa de $\mathrm{rhGH}$, observando-se melhores resultados quando a terapia foi iniciada na fase pré-puberal. Arq Bras Endocrinol Metab. 2010;54(5):443-8

\section{Descritores}

Hormônio de crescimento recombinante; pequeno para idade gestacional; crescimento; preditores clínicos

\begin{abstract}
Objective: To identify clinical predictors associated with catch-up growth in the first year of treatment with somatropin ( $\mathrm{rhGH}$ ) in a group of children born small for gestational age (SGA). Subjects and methods: Thirty nine children who have been on rhGH therapy for at least one year $(0.33 \mathrm{mg} / \mathrm{kg} /$ week) were evaluated. The clinical parameters analyzed were chronological age (CA), bone age (BA), target height and standard deviations scores (SDS) of birth weight and length, height, weight and growth rate. Results: rhGH therapy was associated with a first year height increment of 0.67 SDS $(p<0.01)$. BA and birth weight SDS were predictive of growth response, with statistical difference in height SDS variation between prepubertal and pubertal subgroups $(p=0.016)$. Conclusion: These data show a significant growth response with a fixed rhGH dose, those children who started rhGH in prepubertal ages presented the best outcomes. Arq Bras Endocrinol Metab. 2010;54(5):443-8
\end{abstract}

\section{Keywords}

Recombinant growth hormone; small for gestational age; growth; clinical predictors

Correspondência para: Adriana Claudia Lopes Carvalho Furtado

CCSW 1, lote 1, bloco C, ap. 512, Sudoeste

70680-150 - Brasília, DF, Brasil adrianafurtado@terra.com.br

Recebido em 17/Nov/2009 Aceito em 12/Abr/2010 


\section{INTRODUÇÃO}

$\mathrm{A}$ condição de nascer pequeno para a idade gestacional (PIG) está intimamente relacionada a respostas adaptativas iniciadas no período fetal, que podem se manifestar clinicamente em etapas posteriores da vida, como maior predisposição a desenvolver obesidade visceral, avanço na maturação óssea durante a fase de crescimento e distúrbios metabólicos (1-3). Adicionalmente, crianças nascidas PIG possuem risco aumentado de apresentar baixa estatura na vida adulta (4), risco que chega a ser cinco vezes maior em crianças nascidas PIG para o peso e sete vezes maior em crianças nascidas PIG para o comprimento quando comparadas a crianças nascidas adequadas para a idade gestacional (AIG) $(3,5)$.

Cerca de $10 \%$ a $15 \%$ de todas as crianças nascidas PIG não fazem catch up do crescimento, permanecendo abaixo de -2,0 Z -escore da estatura-alvo. Postula-se que aproximadamente $20 \%$ de todos os adultos com baixa estatura foram PIG ao nascimento (4).

$O$ benefício do tratamento com hormônio de crescimento humano recombinante $(\mathrm{rhGH})$ nessas crianças está consensualmente estabelecido (6). As principais variáveis reconhecidas como preditoras do crescimento do primeiro ano de tratamento são a dose do hormônio utilizada, a idade de início do tratamento, o Z-escore de peso pré-tratamento e o canal familiar $(7,8)$. Contudo, essas variáveis não são capazes de explicar por completo a variabilidade observada no ganho estatural.

Reconhecer os fatores preditores clínicos da resposta ao tratamento com rhGH tem grande importância, uma vez que podem auxiliar na individualização e otimização do manejo terapêutico das crianças nascidas PIG.

Dessa forma, o objetivo deste estudo foi identificar variáveis clínicas relacionadas ao ganho estatural no primeiro ano de tratamento com dose fixa de rhGH em crianças nascidas PIG, acompanhadas no Setor de Endocrinologia do Hospital de Base de Brasília.

\section{SUJEITOS E MÉTODOS}

O estudo foi realizado de acordo com a resolução 196/96 das diretrizes e normas reguladoras de pesquisas envolvendo seres humanos do Conselho Nacional de Saúde e aprovado pelo Comitê de Ética em Pesquisa da Fundação de Ensino e Pesquisa em Ciências da Saúde (FEPECS) do Distrito Federal. O termo de consentimento livre e esclarecido foi obtido dos pais ou responsáveis legais de cada criança.
Trata-se de estudo de coorte clínica observacional retrospectivo, em que foram avaliados os dados do primeiro ano de tratamento de 39 pacientes nascidos PIG cadastrados no Programa de Tratamento com Hormônio de Crescimento (PTGH) da Secretaria de Estado de Saúde do Distrito Federal (SES/DF).

Os critérios de inclusão foram: (a) peso e/ou comprimento ao nascimento igual ou abaixo do percentil 3 (P3) para sexo e idade gestacional (equivalência $\leq-1,88$ desvios-padrão), definidos pela curva populacional de peso e comprimento ao nascimento de Babson Benda (9); (b) estatura prévia ao tratamento abaixo de -2,0 Zescore da curva de crescimento; (c) Z-escore de velocidade de crescimento menor que zero, com o intuito de excluir crianças que estivessem apresentando catch $u p$ de crescimento espontâneo; (d) pacientes nascidos PIG pré-púberes e púberes, com idade cronológica mínima ao início do tratamento de 2 anos para os pacientes PIG nascidos a termo (idade gestacional $\geq 37$ semanas), e de 4 anos para os pacientes PIG pré-termo (idade gestacional $\leq 36$ semanas e 6 dias; (e) idade óssea máxima de 13 anos para meninos e 12 anos para meninas.

Foram excluídos do estudo pacientes nascidos PIG que apresentaram catch up de recuperação do crescimento nos primeiros 2 anos de vida e aqueles portadores de doenças clínicas crônicas.

Os pacientes foram avaliados periodicamente a cada 3 meses e a dose do rhGH utilizada foi $1,0 \mathrm{UI} / \mathrm{kg} /$ semana $(0,33 \mathrm{mg} / \mathrm{kg} /$ semana $)$ administrada em doses diárias. O hormônio de crescimento recombinante utilizado para o tratamento foi o Humatrop ${ }^{\circledR}$ (4 UI por frasco-ampola, Laboratório Bergamo).

A idade óssea foi determinada pelo método de Greulich-Pyle (10). Pacientes que apresentaram atraso de idade óssea maior que 2 anos foram submetidos a teste de estímulo do GH com clonidina e/ou insulina para afastar possível deficiência hormonal, tendo como ponto de corte $7,0 \mathrm{ng} / \mathrm{ml}$ (método quimioluminescência).

A velocidade de crescimento pré-tratamento foi determinada pelo intervalo de acompanhamento de, no mínimo, 10 meses e, no máximo, 14 meses.

As variáveis comprimento e peso ao nascer, altura e peso pré e 1 ano pós-tratamento, velocidade de crescimento e ganho estatural foram expressas em escore de desvio-padrão (Z-escore). Os Z-escore de peso e comprimento ao nascimento foram calculados com base na curva de crescimento intrauterino construída por Margotto (11), que é uma curva local do Distrito Federal. A simetria do recém-nascido ( $\mathrm{RN})$ foi avaliada pelo 
índice ponderal: IP $=$ [peso em gramas $/$ comprimento em $\mathrm{cm})^{3}$ ] x 100, definindo o RN como simétrico (ou Tipo I) e assimétrico (Tipo II) (12).

Os dados auxológicos dos pacientes foram analisados por meio do programa Growth Analyser versão 3.5 (Dutch Growth Foundation, Rotterdan, The Netherlands), utilizando-se para cálculo do Z-escore de estatura e peso e estatura-alvo a curva do NCHS 2000 e para cálculo do Z-escore de velocidade de crescimento a curva de Tanner (1976) contidas no programa. A estatura-alvo foi calculada a partir da fórmula: altura do pai + altura da mãe + 13 (meninos) ou - 13 (meninas) $\div 2(13)$.

O ganho estatural ( $\Delta \mathrm{Z}$-escore de estatura) após 1 ano de tratamento, medido pela diferença entre o Z-escore da estatura após 1 ano de tratamento e o Z-escore da estatura inicial, foi a variável de desfecho escolhida para avaliar o efeito do tratamento com rhGH.

No que se refere à análise estatística, primeiramente foi feita avaliação da normalidade das variáveis estudadas pelo teste de Kolmogorov-Smirnov. Como essa avaliação apontou para uma distribuição normal, a avaliação subsequente se deu com a utilização do teste $t$ de Student para comparação das médias da variável desfecho em relação às variáveis independentes sexo, idade gestacional e simetria antropométrica. Para comparação entre os subgrupos pré-púbere, púbere e púbere em bloqueio puberal com triptorrelina, assim como dos subgrupos de crianças nascidas PIG para peso, PIG para comprimento e PIG para peso e comprimento, utilizou-se o teste ANOVA de uma via, sendo utilizada a correção de Tukey para as comparações post hoc.

Para identificar as variáveis preditoras do ganho estatural no primeiro ano de tratamento, analisou-se o $\Delta \mathrm{Z}$-escore de estatura em um modelo de regressão linear múltipla passo a passo com as variáveis independentes: idade cronológica, idade óssea, Z-escore de peso e estatura ao nascimento, Z-escore de peso e estatura no início do tratamento e Z-escore de estaturaalvo. Para entrada no modelo de regressão, utilizou-se como critério a razão $\mathrm{F}$ maior ou igual a 3,84 , o que corresponde a uma probabilidade $\mathrm{p}$ associada menor ou igual a 0,05. Como critério de remoção da variável no modelo, utilizou-se uma razão $\mathrm{F}$ menor ou igual a 2,71, o que corresponde a uma probabilidade $\mathrm{p}$ maior ou igual a 0,10 .

O nível de significância estabelecido foi de $5 \%$. As análises estatísticas foram realizadas usando o programa SPSS versão 17.0 para Windows.

\section{RESULTADOS}

Entre os 39 pacientes, 23 eram do sexo masculino e, ao nascimento, 29 foram simétricos e 27 nasceram a termo. No início da terapia com rhGH, 24 pacientes eram pré-púberes e 15 eram púberes, sendo que, entre esses últimos, 6 pacientes iniciaram o rhGH associado ao bloqueio puberal com triptorrelina $(3,75 \mathrm{mg}$ intramuscular de 28 em 28 dias) com objetivo de prolongar o tempo de crescimento e otimizar a resposta ao rhGH. Do total, 7 pacientes nasceram PIG para peso, 17 eram PIG para comprimento e 15 eram PIG para peso e comprimento.

As características clínicas dos pacientes estão apresentadas na tabela 1 .

Tabela 1. Características auxológicas dos pacientes ao nascimento e no início do tratamento com rhGH

\begin{tabular}{lcccc}
\hline Variáveis & Mediana & $\begin{array}{c}\text { Percentis } \\
\mathbf{1 0 - 9 0}\end{array}$ & Média & DP \\
\hline IG (semanas) & 38,00 & 33 a 40 & 37,23 & 2,78 \\
IC (anos) & 9,83 & 3,75 a 14,1 & 9,41 & 3,54 \\
I0 (anos) & 7,83 & 2,00 a 12,2 & 7,61 & 3,69 \\
Z-escore peso nasc. & $-1,90$ & $-2,75$ a $-0,80$ & $-1,81$ & 0,66 \\
Z-escore comp. nasc. & $-1,90$ & $-3,12$ a $-0,20$ & $-1,83$ & 0,90 \\
Z-escore estatura inicial & $-2,78$ & $-3,82$ a - 2,12 & $-2,88$ & 0,62 \\
Z-escore IMC & $-1,16$ & $-2,77$ a 0,30 & $-1,16$ & 1,15 \\
Z-escore peso inicial & $-2,64$ & $-4,31$ a $-1,39$ & $-2,92$ & 1,43 \\
Z-escore VC inicial & $-1,18$ & $-2,62$ a $-0,34$ & $-1,38$ & 1,00 \\
Z-escore estatura-alvo & $-0,54$ & $-1,67$ a 0,23 & $-0,58$ & 0,70 \\
\hline
\end{tabular}

IG: idade gestacional; IC: idade cronológica; IO: idade óssea; IMC: índice de massa corpórea; VC: velocidade de crescimento; nasc. (ao nascer); comp. (comprimento); DP: desvio-padrão.

O impacto do tratamento com rhGH no Z-escore de estatura, na velocidade de crescimento e no Z-escore da velocidade de crescimento é mostrado na tabela 2 .

Tabela 2. Resposta ao tratamento com rhGH após 1 ano no grupo total de pacientes $(n=39)$

\begin{tabular}{lcccc}
\hline & Antes do rhGH & $\begin{array}{c}\text { 1 ano após } \\
\text { rhGH }\end{array}$ & $\Delta$ & p \\
\hline $\begin{array}{l}\text { Z-escore estatura } \\
\text { Média (DP) }\end{array}$ & $-2,89(0,62)$ & $-2,21(0,70)$ & 0,67 & $<0,01$ \\
$\begin{array}{l}\text { VC } \\
\text { Média (DP) }\end{array}$ & $4,96(1,16)$ & $9,19(2,05)$ & 4,20 & 0,10 \\
$\begin{array}{l}\text { Z-escore VC } \\
\text { Média (DP) }\end{array}$ & $-1,38(1,00)$ & $3,55(2,35)$ & 4,93 & 0,91 \\
\hline
\end{tabular}

rhGH: hormônio de crescimento recombinante humano; $\Delta$ : diferença entre os valores após 1 ano e pré-rhGH; VC: velocidade de crescimento; DP: desvio-padrão.

A idade óssea e a idade cronológica apresentaram correlação inversa com o ganho estatural e com a velocidade de crescimento após 1 ano de tratamento. Observou-se correlação direta entre peso e Z-escore 
da estatura inicial e de $\mathrm{l}$ ano após rhGH. Os Z-escore de comprimento ao nascimento e de estatura-alvo não apresentaram correlação significativa com as variáveis independentes analisadas.

Duas variáveis foram identificadas como preditoras do ganho estatural do primeiro ano de tratamento: idade óssea $(\mathrm{p}<0,001)$ e Z-escore de peso ao nascimento $(\mathrm{p}=0,039)$. Associadas, ambas foram responsáveis por um coeficiente de correlação geral $\left(\mathrm{R}_{2}\right)$ de 0,417 , capazes de explicar cerca de $42 \%$ da variabilidade do ganho estatural.

O coeficiente B estabeleceu que a cada ano de avanço da idade óssea há uma perda de 0,62 Z-escore no ganho estatural e cada aumento no Z-escore de peso ao nascimento corresponde a um incremento de $0,27 \mathrm{Z}$-escore no ganho estatural do primeiro ano de tratamento.

As diferenças no ganho estatural após 1 ano de tratamento entre os subgrupos, classificados de acordo com as características clínicas estudadas, são mostradas na tabela 3. Nessa avaliação, observou-se diferença estatisticamente significativa apenas entre os grupos de pacientes púberes e pré-púberes $(\mathrm{p}=0,01)$.

Tabela 3. Diferenças no ganho estatural entre os subgrupos das crianças nascidas PIG

\begin{tabular}{lcc}
\hline Grupos & $\begin{array}{c}\Delta \text { Z-escore estatura } \\
\text { (média) }\end{array}$ & Valor $\mathbf{p}$ \\
\hline Masculino/Feminino & $0,69 / 0,64$ & 0,68 \\
Pré-termo/Termo & $0,66 / 0,67$ & 0,91 \\
PIG simétrico/assimétrico & $0,70 / 0,57$ & 0,35 \\
Pré-púbere/Púbere & $0,82 / 0,40$ & 0,01 \\
Pré-púbere/Púbere bloqueado* $^{*}$ & $0,82 / 0,47$ & 0,08 \\
Púbere/Púbere bloqueado* $^{*}$ & $0,47 / 0,40$ & 0,92 \\
\hline
\end{tabular}

* Púbere bloqueado: grupo de pacientes em bloqueio puberal com triptorrelina. PIG: pequeno para a idade gestacional.

As médias de Z-escore de ganho estatural entre os subgrupos de crianças nascidas PIG para peso, PIG para comprimento e PIG para peso e comprimento foram de $0,55,0,72$ e 0,66 respectivamente, sem diferença estatisticamente significativa entre esses subgrupos $(p=0,63)$.

\section{DISCUSSÃO}

Apesar de alguns estudos demonstrarem relação direta entre dose e resposta ao tratamento nas crianças nascidas PIG $(7,8,14,15)$, nosso estudo mostrou que a dose fixa de $1,0 \mathrm{UI} / \mathrm{kg} / \mathrm{semana}(0,33 \mathrm{mg} / \mathrm{kg} / \mathrm{semana})$ foi capaz de promover um catch up de crescimento rele- vante no primeiro ano de tratamento, constatado pela diferença significativa de $0,67 \mathrm{Z}$-escore no ganho estatural nesse período.

A observação de respostas de crescimento tão variadas a diferentes doses de rhGH na literatura sugere que outros fatores possam influenciar na resposta individual. Em estudo comparativo entre dose individualizada $(0,035 \mathrm{mg} / \mathrm{kg} /$ dia inicialmente $)$ e dose fixa $(0,067$ $\mathrm{mg} / \mathrm{kg} /$ dia) de rhGH, Jung e cols. (16) demonstraram que a dose individualizada mais baixa não foi inferior na promoção do crescimento do primeiro ano de tratamento de crianças PIG, constatando que a dose mais alta não foi necessária na metade das crianças tratadas. Essa observação pode minimizar o custo da prescrição de doses iniciais até duas vezes maiores, principalmente nos casos de instituições públicas de saúde financiarem o tratamento dessas crianças.

Nesse estudo, apesar da observação de ganho estatural significativo com o uso do rhGH ( $\mathrm{p}<0,01)$, a análise estatística não mostrou diferença significativa no $\Delta$ de velocidade de crescimento do primeiro ano de tratamento $(\mathrm{p}=0,91)$. Esse fato pode ser justificado pela média de 9,4 anos de idade cronológica dos pacientes e pela presença de pacientes púberes no estudo, uma vez que uma melhor resposta terapêutica é observada quanto mais precoce for instituído o tratamento (17).

A resposta no primeiro ano de tratamento não mostrou correlação com os Z-escore da estatura inicial e da estatura-alvo, os quais são citados na literatura como variáveis clínicas preditoras de ganho estatural em fases subsequentes do tratamento, como no início da puberdade e na estatura final $(18,19)$.

A idade óssea no início do tratamento, em acordo com a literatura $(7,20)$, foi identificada como uma das variáveis preditoras do ganho estatural no primeiro ano de tratamento, ressaltando a correlação negativa com o ganho estatural (2l).

Contudo, o Z-escore de peso ao nascimento não é citado na literatura como variável preditora da resposta no primeiro ano de tratamento. Acreditamos que a presença de crianças nascidas prematuras e PIG, com baixo peso ao nascimento, na amostra estudada, influenciou o surgimento dessa variável como fator preditor, uma vez que a restrição no crescimento intra-hospitalar dessas crianças (22), com papel dominante sobre o eixo GHIGF-I (23), estaria relacionada a processos de reprogramação nutricional pós-natal, levando a alterações metabólicas similares àquelas observadas em $\mathrm{RNs}$ nascidos a termo PIG (24). 
Não houve diferença na resposta ao rhGH entre pacientes PIG nascidos pré-termo e termo, o que está de acordo com o descrito por alguns autores, os quais relacionam a prematuridade a um fenótipo mais longilíneo na infầncia, mas com resposta ao tratamento hormonal semelhante ao das crianças nascidas a termo (25).

Comparando-se o ganho estatural entre os subgrupos de pacientes pré-púberes, púberes e púberes em bloqueio puberal, observou-se um ganho significativamente maior nos pacientes pré-púberes em relação aos pacientes púberes $(\mathrm{p}=0,01)$. Tal observação pode estar relacionada a uma maior resistência ao hormônio de crescimento durante a puberdade, quando os pacientes púberes necessitariam de uma dose maior do rhGH para otimizar o crescimento linear.

A avaliação da ausência de diferenças estatisticamente significantes entre o grupo púbere em bloqueio puberal com os grupos pré-púbere e púbere fica limitada pelo número pequeno de pacientes nos referidos grupos. Contudo, observa-se tendência de resposta de ganho estatural do grupo púbere bloqueado mais próxima do grupo púbere $(\mathrm{p}=0,08)$ do que do grupo prépubere $(\mathrm{p}=0,92)$. Entretanto, há necessidade de estudos posteriores com um número maior de pacientes para uma avaliação mais adequada quanto ao benefício do bloqueio puberal associado ao uso do rhGH sobre a estatura final dessas crianças.

Quando se avaliou o ganho estatural nos subgrupos divididos pelo índice ponderal, e entre os nascidos PIG para peso, PIG para comprimento e PIG para peso e comprimento, não foi observada diferença na resposta ao tratamento com rhGH. Essa ausência de diferentes respostas também é observada por alguns autores (26), recomendando-se a utilização dessas subclassificações apenas para investigação etiológica, uma vez que estariam relacionadas a condições de gestação e poderiam influenciar o tipo de parto e de crescimento pós-natal (27).

Em conclusão, a dose fixa do rhGH de $1,0 \mathrm{UI} / \mathrm{kg} /$ semana foi capaz de promover um catch up de crescimento significativo nesse grupo de pacientes nascidos PIG. As variáveis clínicas identificadas como preditoras do ganho estatural do primeiro ano de tratamento foram a idade óssea e o Z-escore de peso ao nascimento. Contudo, quando associados, estes foram capazes de explicar apenas $42 \%$ da variabilidade de crescimento, reforçando a necessidade da busca continuada de outros possíveis fatores preditores. A comparação dos subgrupos classificados quanto ao estadiamento puberal mos- trou que a melhor resposta ao rhGH é obtida quanto mais precoce for instituído o tratamento.

Agradecimentos: ao Programa de Tratamento com Hormônio de Crescimento da Secretaria de Saúde do Distrito Federal e ao valioso apoio da Dra. Margaret C. S. Boguszewski.

Declaração: os autores declaram não haver conflitos de interesse científico neste estudo.

\section{REFERÊNCIAS}

1. Gluckman PD, Hanson MA. The consequences of being born small - an adaptive perspective. Horm Res. 2006;65 Suppl 3:5-14.

2. Wollmann HA. Intrauterine growth restriction: definition and etiology. Horm Res. 1998;49 Suppl 2:1-6.

3. Albertsson-Wikland K, Boguszewski M, Karlberg J. Children born small-for-gestational age: postnatal growth and hormonal status. Horm Res. 1998;49 Suppl 2:7-13.

4. Karlberg J, Albertsson-Wikland K. Growth in full-term small-forgestational-age infants: from birth to final height. Pediatr Res. 1995;38(5):733-9.

5. Lee PA, Kendig JW, Kerrigan JR. Persistent short stature, other potential outcomes, and the effect of growth hormone treatment in children who are born small for gestational age. Pediatrics. 2003;112(1 Pt 1):150-62.

6. Clayton PE, Cianfarani S, Czernichow P, Johannsson G, Rapaport R, Rogol A. Management of the child born small for gestational age through to adulthood: a consensus statement of the International Societies of Pediatric Endocrinology and the Growth Hormone Research Society. J Clin Endocrinol Metab. 2007;92(3):804-10.

7. Ranke MB, Lindberg A, Cowell CT, Wikland KA, Reiter EO, Wilton $P$, et al. Prediction of response to growth hormone treatment in short children born small for gestational age: analysis of data from KIGS (Pharmacia International Growth Database). J Clin Endocrinol Metab. 2003;88(1):125-31.

8. Boguszewski M, Albertsson-Wikland K, Aronsson S, Gustafsson J, Hagenas L, Westgren $U$, et al. Growth hormone treatment of short children born small-for-gestational-age: the Nordic Multicentre Trial. Acta Paediatr. 1998;87(3):257-63.

9. Fenton TR. A new growth chart for preterm babies: Babson and Benda's chart updated with recent data and a new format. BMC Pediatr. 2003;3:13.

10. Greulich WW, Pyle SI. Radiographic atlas of skeletal development of the hand and wrist. 2nd ed. Stanford University Press: Stanford; 1959.

11. Margotto PR. [Intrauterine growth curves: study of 4413 single live births of normal pregnancies]. J Pediatr. 1995;71(1):11-21.

12. Pescador MVB, Streher AAF, Silva JMF. Aspectos endocrinológicos das crianças e adultos nascidos pequenos para a idade gestacional. Arq Bras Endocrinol Metab. 2001;1(45):15-23.

13. Kochi $C$, Longui $C A$. Critérios de avaliação do crescimento normal. In: Kochi C, Longui CA, editores. Endocrinologia para o pediatra. São Paulo: Atheneu; 2006. p. 31-6.

14. Crabbe R, von Holtey M, Engrand P, Chatelain P. Recombinant human growth hormone for children born small for gestational age: meta-analysis confirms the consistent dose-effect relationship on catch-up growth. J Endocrinol Invest. 2008;31(4):346-51.

15. Tanaka T, Fujieda K, Yokoya S, Seino Y, Tada H, Mishina J. Efficacy and safety of growth hormone treatment in children born small for gestational age in Japan. J Pediatr Endocrinol Metab. 2008;21(5):423-31. 
16. Jung H, Land C, Nicolay C, De Schepper J, Blum WF, Schonau E. Growth response to an individualized versus fixed dose $\mathrm{GH}$ treatment in short children born small for gestational age: the OPTIMA study. Eur J Endocrinol. 2009;160(2):149-56.

17. de Zegher $F$, Butenandt $O$, Chatelain $P$, Albertsson-Wikland $K$, Jonsson B, Lofstrom A, et al. Growth hormone treatment of short children born small for gestational age: reappraisal of the rate of bone maturation over 2 years and metanalysis of height gain over 4 years. Acta Paediatr Suppl. 1997;423:207-12.

18. de Ridder MA, Stijnen T, Hokken-Koelega AC. Prediction model for adult height of small for gestational age children at the start of growth hormone treatment. J Clin Endocrinol Metab. 2008;93(2):477-83.

19. Van Pareren Y, Mulder P, Houdijk M, Jansen M, Reeser M, HokkenKoelega A. Adult height after long-term, continuous growth hormone $(\mathrm{GH})$ treatment in short children born small for gestational age: results of a randomized, double-blind, dose-response $\mathrm{GH}$ trial. J Clin Endocrinol Metab. 2003;88(8):3584-90.

20. Rapaport R, Saenger P, Wajnrajch MP. Predictors of first-year growth response to a fixed-dose growth hormone treatment in children born small for gestational age: results of an open-label, multicenter trial in the United States. J Pediatr Endocrinol Metab. 2008;21(5):411-22.

21. Sas T, de Waal W, Mulder P, Houdijk M, Jansen M, Reeser M, et al. Growth hormone treatment in children with short stature born small for gestational age: 5-year results of a randomized, double-blind, dose-response trial. J Clin Endocrinol Metab. 1999;84(9):3064-70.

22. Lemons JA, Bauer CR, Oh W, Korones SB, Papile LA, Stoll BJ, et al. Very low birth weight outcomes of the National Institute of Child health and human development neonatal research network, January 1995 through December 1996. NICHD Neonatal Research Network. Pediatrics. 2001;107(1):E1.

23. Cutfield WS, Regan FA, Jackson WE, Jefferies CA, Robinson EM, Harris $M$, et al. The endocrine consequences for very low birth weight premature infants. Growth Horm IGF Res. 2004;14 Suppl A:S130-5.

24. Hofman PL, Regan F, Harris M, Robinson E, Jackson W, Cutfield WS. The metabolic consequences of prematurity. Growth Horm IGF Res. 2004;14 Suppl A:S136-9.

25. de Kort SW, Willemsen RH, van der Kaay DC, Duivenvoorden HJ, Hokken-Koelega AC. Does preterm birth influence the response to growth hormone treatment in short, small for gestational age children? Clin Endocrinol (Oxf). 2009;70(4):582-7.

26. Mehls O, Lindberg A, Bettendorf M, Doerr HG, Hauffa BP, Kaspers $\mathrm{S}$, et al. Is the response to growth hormone in short children born small for gestational age dependent on genetic or maternal factors? Horm Res. 2009;72(2):106-13.

27. Ester W, Bannink E, van Dijk M, Willemsen R, van der Kaay D, de Ridder M, et al. Subclassification of small for gestational age children with persistent short stature: growth patterns and response to GH treatment. Horm Res. 2008;69(2):89-98. 